\title{
In reply: Use of low-molecular weight dextran as an LA adjuvant to extend LA action
}

\author{
Masaru Tobe $^{1}$ (D) Shigeru Saito ${ }^{1}$
}

Received: 26 October 2018 / Accepted: 27 October 2018 / Published online: 5 December 2018

(c) Japanese Society of Anesthesiologists 2018

Keywords Local anesthetics (LA) $\cdot$ Dextran $\cdot$ Extend LA action

To the Editor,

We sincerely thank Dr. Tsuchiya and colleagues for their interest and comments on our manuscript. We presented the historical sequences of studies that have been performed in an effort to elongate the action of local anesthetics (LA) in past review [1]. We did not describe the use of dextran as an LA adjuvant in this review, but it is interesting to extend the LA action using with low-molecular weight dextran. Hamada et al. demonstrated that the absorption of levobupivacaine was suppress in 90 min notably adding the $8 \%$ lowmolecular weight dextran solution [2]. We hope for high quality postoperative pain management using long acting
LA. In this view, we expect the large volume clinical data with novel technique or novel drug.

\section{References}

1. Tobe M, Suto T, Saito S. The history and progress of local anesthesia: multiple approaches to elongate the action. J Anesth. 2018;32:632-6.

2. Hamada T, Tsuchiya M, Mizutani K, et al. Levobupivacaine-dextran mixture for transversus abdominis plane block and rectus sheath block in patients undergoing laparoscopic colectomy: a randomised controlled trial. Anaesthesia. 2016;71:411-6.

This reply refers to the comment available online at https://doi. org/10.1007/s00540-018-2573-x.

Masaru Tobe

to-be@f3.dion.ne.jp

1 Department of Anesthesiology, School of Medicine,

Gunma University Graduate, 3-39-22, Showa, Maebashi,

Gunma 371-8511, Japan 